\title{
Genetic Association of Apolipoprotein A5-1131T>C Polymorphism with Traits of Metabolic Syndrome
}

\author{
Uzma Zafar, Saba Khaliq and Khalid Pervaiz Lone
}

\begin{abstract}
Objective: To investigate the association of $-1131 \mathrm{~T}>\mathrm{C}$ polymorphism of apolipoprotein A5 (APOA5) with metabolic syndrome and associated traits.

Study Design: A cross-sectional comparative study.

Place and Duration of Study: Department of Physiology, University of Health Sciences Lahore, from July 2016 to December 2017. Methodology: Study population included 200 cases of metabolic syndrome and 200 controls. Cases were selected from Sheikh Zayed Hospital, Lahore according to the International Diabetes Federation guidelines. Fasting blood sample of $8 \mathrm{ml}$ was taken for biochemical and genetic analysis, as per objective. Demographics, waist circumferance and blood pressure (BP) were also recorded.

Results: Subjects with metabolic syndrome had significantly higher waist circumference, BP, serum lipid and glycemic parameters as compared to the controls $(p<0.001)$. The observed frequency of APOA5-1131T>C genotype TT was 180 $(45 \%), 202(50.5 \%)$ for TC and $18(4.5 \%)$ for CC. Minor C allele frequency of APOA5-1131T>C variant was significantly higher in metabolic syndrome as compared to the controls $\left(0.33\right.$ vs. $\left.0.26: p=0.031^{*}\right)$. In the Dominant genotype model $(\mathrm{TC}+\mathrm{CC}$ vs. TT), 'TC+CC' genotype was significantly associated with the increased risk of metabolic syndrome (OR: 1.50, $\left.\mathrm{Cl}: 1.01-2.23, \mathrm{p}=0.044^{*}\right)$. Waist circumference and fasting triglyceride levels were significantly higher in 'TC $+\mathrm{CC}$ ' genotype as compared to the 'TT' in metabolic syndrome and controls $(p=<0.001$ and $0.043 ; p=0.022$ and 0.006$)$.

Conclusion: There was significant association of the 'TC+CC' genotype of APOA5 $-1131 T>C$ with the increased risk of Met $\mathrm{S}$ and the association remained significant after controlling for age and gender.
\end{abstract}

Key Words: Metabolic syndrome, Single nucleotide polymorphism, -1131T>C promoter variant, Apolipoprotein A5, Lipid profile.

\section{INTRODUCTION}

Metabolic syndrome (Met S) is a cluster of clinical and biochemical abnormalities such as central obesity hypertension, hyperglycemia, insulin resistance and dyslipidemia. 1 These metabolic derangements significantly increase the risk of coronary artery disease (CAD), stroke and hepatic steatosis. ${ }^{2}$ Met $S$ is a global health issue of the recent times. According to the International Diabetes Federation (IDF) definition its prevalence in Pakistan is reported to be $35 \%$ and females are affected more than the males with the prevalence of $36 \%$ and $31 \%$, respectively. ${ }^{3}$ Besides acquired risk factors such as excessive caloric intake and lack of physical activity, genetic variability plays a key role in predisposition to insulin resistance and Met $S$. Various genome wide association studies (GWAS) and candidate gene sequencing have identified certain susceptibility loci for insulin resistance and Met S. 4 Single nucleotide polymorphisms (SNP) of apolipoprotein A5 (APOA5) gene have been reported to be associated with the increased susceptibility to the Met $S$ and the

Department of Physiology, University of Health Sciences,

Lahore, Pakistan

Correspondence: Dr. Uzma Zafar, Department of Physiology,

University of Health Sciences, Lahore, Pakistan

E-mail:uzma.zargham@gmail.com

Received: October 03, 2018; Revised: December 29, 2018;

Accepted: January 08, 2019 related traits. The APOA5 gene is located within the APOA1/C3/A4/A5 gene cluster on chromosome 11q23 and consists of 4 exons encoding APOA5 a 369 amino acid protein. APOA5 is synthesized primarily in the liver and plays a major role in the regulation of the triglyceride metabolism. The plasma concentration of APOA5 is very small $(100 \mu \mathrm{g} / \mathrm{L})$ and it is mainly present on the surface of triglyceride particles such as very low density lipoproteins (VLDL), high density lipoproteins (HDL) and chylomicrons. ${ }^{5} \mathrm{~A}$ higher plasma APOA5 results in low blood triglycerides and negative correlation has been reported between plasma triglyceride and APOA5 levels. ${ }^{6}$ APOA5 stimulates the enzyme lipoprotein lipase that lowers plasma triglycerides by increasing the hydrolysis of triglycerides from the core of chylomicrons and VLDL into fatty acids and glycerol, thus generating intermediate density lipoproteins from VLDL and chylomicron remnants. APOA5 also inhibits the formation of VLDL-triglycerides and modulates the metabolism of lipids other than the triglycerides such as cholesterol and HDL-cholesterol. Several studies have demonstrated the association of APOA5 polymorphism with HDLcholesterol and triglycerides in the blood.7,8 Promoter region polymorphism of APOA5 $-1131 \mathrm{~T}>\mathrm{C}$ was reported to be associated with raised serum triglycerides and the risk of coronary artery disease in various ethnic groups such as Africans, Spaniards and Caucasians. ${ }^{9}$ In Puerto Ricans, this SNP was not associated with the metabolic lipid profile. ${ }^{10}$ Genetic variant $-1131 \mathrm{~T}>\mathrm{C}$ (rs662977) of 
APOA5 is extensively studied for the possible association with the blood lipids but the results are equivocal and conflicting.

Various studies have reported ethnic specific association of this polymorphism with Met $S$ and related traits. 5 In Pakistan, previous two studies have reported the association of APOA5 -1131 T>C polymorphism with CAD and blood lipid profile. However, available data is sparse regarding the association of this genetic variant with Met S.11,12 The present study was designed to determine the frequency of APOA5 -1131 T>C polymorphism in Met $S$ and healthy group and to associate it with Met $S$ related traits included in the selection of the subjects as per IDF guidelines.

\section{METHODOLOGY}

This cross-sectional comparative study was conducted in the Department of Physiology and Cell Biology, University of Health Sciences, Lahore from July 2016 to December 2017. It was approved by the Institutional Review Board of the University. All participants were fully informed of the study and written informed consent was taken. Study population included 200 cases of Met S and 200 subjects without Met S. Cases of Met S were recruited from Endocrinology and Diabetic Clinic of Sheikh Zayed Hospital, Lahore. A questionnaire was used to gather the data on disease history, demographics, clinical and biochemical para-meters and medications. Met $S$ was defined as per guidelines of the IDF.13 All the selected cases of Met $S$ were centrally obese; males having waist circumference $\geq 90 \mathrm{~cm}$ and females having waist circumference $\geq 80 \mathrm{~cm}$ with serum triglycerides $\geq 150 \mathrm{mg} / \mathrm{dl}$ or on lipid lowering agents, serum HDL-c $<40 \mathrm{mg} / \mathrm{dl}$ for men and less than $50 \mathrm{mg} / \mathrm{dl}$ for women or on treatment for dyslipidemia, fasting blood sugar $>100 \mathrm{mg} / \mathrm{dl}$ or on treatment for diabetes mellitus, and blood pressure $>130 / 85 \mathrm{mmHg}$ or on treatment for hypertension. If two of the above four features were present along with the central obesity, subjects were diagnosed to have Met $\mathrm{S}$. In order to take fasting blood sample status of the subjects, the fasting was defined as no caloric intake for the last 8 to 10 hours. ${ }^{14}$ All those having evidence of acute infection, hepatic or renal decompensation, secondary causes of diabetes mellitus, chronic inflammatory or infective conditions were excluded. Age and gender matched controls were selected from the hospitals. They were hospital staff or non blood related attendants of the out patients. Selection criteria for the controls was: waist circumference $<90 \mathrm{~cm}$ for men and $<80 \mathrm{~cm}$ for women, non diabetic and non hypertensive. ${ }^{13}$

Blood pressure and waist circumference were measured by the standard methods. ${ }^{15} \mathrm{~A}$ fasting venous blood sample of $8 \mathrm{ml}$ was taken and secured for DNA extraction and measurement of biochemical parameters. Fasting serum glucose, triglycerides, HDL-c were measured with spectrophotometer by Colorimetric method (Randox Kit, United Kingdom). Insulin was measured by Human Insulin ELISA Kit (ElabScience, Germany). Insulin resistance was calculated from serum glucose $(\mathrm{mmol} / \mathrm{L})$ and fasting serum insulin $(\mu \mathrm{IU} / \mathrm{ml})$ by HOMA-IR (homeostatic model assessment for insulin resistance) using the equation, HOMA-IR=Fasting glucose $(\mathrm{mmol} / \mathrm{L}) X$ fasting insulin $(\mu \mathrm{IU} / \mathrm{ml}) / 22.5 .16$

DNA was extracted by blood genomic DNA extraction kit FavorPrep (Favorgen,Taiwan, China) according to the manufacturer's guidelines. DNA yield was checked by the nanodrop. Genotyping of APOA5 -1131 T>C rs662799 genetic variant was done by polymerase chain reaction (PCR) followed by the restriction fragment length polymorphism (RFLP).

The forward and backward primer sequence used were: 5'-GATTGATTCAAGATGCATTTAGGAC-3' and 5'-CCCC AGGAACTGGAGCGAAATT-3'. Each PCR reaction mixture comprised of 100ng (almost $2 \mu \mathrm{L}$ ) of DNA, 0.8 $\mu \mathrm{L}$ of both primers (10 pmol/ $\mu \mathrm{L}$ each), $8 \mu \mathrm{L}$ master mix and $5 \mu \mathrm{L}$ of distilled water. For the product amplification, initial denaturation was carried at $95^{\circ} \mathrm{C}$ for 5 mins, followed by 30 cycles of denaturation at $95^{\circ} \mathrm{C}$ for $15 \mathrm{~s}$, annealing at $61^{\circ} \mathrm{C}$ for $1 \mathrm{~min}$, and extension at $72^{\circ} \mathrm{C}$ for 1 min, with final extension at $72^{\circ} \mathrm{C}$ for 7 mins. The restriction enzyme used was Tru1l or Mse1 and restriction reaction was performed in $15 \mu \mathrm{L}$ amplified PCR mixture along with $2 \mu \mathrm{L}$ buffer and $3 \mathrm{U}$ of restriction enzyme with 12 hour incubation at $37^{\circ} \mathrm{C}$. Products of digestion were separated and identified on $3 \%$ agarose gel by electrophoresis. Three genotypes identified were: TT (wild type) 165- and 23- base pair: CC (minor type) 188 base pair: TC (heterozygous) 188-, 165- and 23-base pair (Figures 1 and 2). ${ }^{17}$

Data was entered and analysed by SPSS version 22 . Normal distribution of the data was checked by ShapiroWilk statistics and if $p$-value was less than 0.05 , data was considered to be non-normally distributed. Frequencies and percentages were given for categorical variables. Mean $\pm S D$ and median with interquartile range (IQR) were given for normally and non-normally distributed quantitative variables. Normally distributed quantitative variables were compared by independentsamples t-test and non-normally distributed quantitative variables were compared by Mann-Whitney U-test. genotypic and allelic frequencies of APOA5 -1131 T>C were calculated and Hardy-Weinberg equilibrium was determined by online genetic epidemiology tool (OEGE) (http/www.oege.org). Allelic frequencies of two groups were compared by the online MedCalc and QuickCalcsGraphPad statistical software (http://www.graphpad.com/ quickcalcs/). In order to study the association of genotypes with Met $S$, three genetic models of APOA51131T>C: Co-dominant (TT: TC: CC), Dominant 
(TC+CC vs. TT) and Recessive (CC vs. TC+TT) were constructed. Genotype frequencies of two groups were compared by Chi-square test and odds ratio was calculated. Logistic regression was applied to see the association of genotypes with Met $\mathrm{S}$ after controlling for age and gender.

Table I: Comparison of clinical and biochemical parameters of the study groups.

\begin{tabular}{|c|c|c|c|}
\hline Study parameters & $\begin{array}{c}\text { Metabolic } \\
\text { syndrome } \\
\mathrm{N}=200\end{array}$ & $\begin{array}{l}\text { Healthy } \\
\text { group } \\
\mathrm{N}=200\end{array}$ & $p$-value \\
\hline Age & $46.92 \pm 8.06$ & $46.80 \pm 8.04$ & 0.877 \\
\hline Systolic BP in mm of mercury & $130(110-130)$ & $110(90-120)$ & $<0.001^{*}$ \\
\hline Diastolic BP in mm of mercury & $80(70-90)$ & $75(70-80)$ & $<0.001^{*}$ \\
\hline Waist circumference in $\mathrm{cm}$ & $102(95-106)$ & $80(73-86)$ & $<0.001^{*}$ \\
\hline Serum high density lipoprotein in $\mathrm{mg} / \mathrm{dl}$ & $39(32-44)$ & $39(37-42)$ & 0.948 \\
\hline Serum triglycerides in $\mathrm{mg} / \mathrm{dl}$ & $188(141-271)$ & $146(122-165)$ & $<0.001^{*}$ \\
\hline Serum glucose in $\mathrm{mmol} / \mathrm{L}$ & $8.13(6.59-10.92)$ & $5.01(4.4-5.4)$ & $<0.001^{*}$ \\
\hline Serum insulin in $\mu \mathrm{IU} / \mathrm{L}$ & $28(16.8-47.5)$ & $8.75(6.47-13.5)$ & $<0.001^{*}$ \\
\hline HOMA-IR & $10.52(5.8-17.6)$ & $2.0(1.14-3.14)$ & $<0.001^{*}$ \\
\hline
\end{tabular}

Values given are Mean $\pm S D$ or Median (IQR) as related to the results obtained through the Shapiro-Wilk's statistics. " $p$ " value is generated by "Independent samples $T$ test" for age and by "Mann Whitney U" test for all other parameters. A p of less than 0.05 is statistically significant. HOMA-IR = Fasting serum glucose $\times$ Fasting serum insulin $/ 22.5$.

Table II: Comparison of APOA5 -1131T>C (rs662799) in the study groups.

\begin{tabular}{l|c|c|c|c}
\hline Genotype & $\begin{array}{c}\text { Metabolic } \\
\text { syndrome } \\
\mathrm{n}(\% \text { age) }\end{array}$ & $\begin{array}{c}\text { Healthy } \\
\text { group } \\
\mathrm{n}(\% \text { age })\end{array}$ & $\mathrm{p}$-value & OR and Cl \\
\hline Co-dominant model & $80(40)$ & $100(50)$ & $0.039^{*}$ & OR not computed \\
TT & $107(54)$ & $95(48)$ & & \\
TC & $13(6)$ & $5(2)$ & & \\
CC & 200 & 200 & & \\
Total & $133(33)$ & $105(26)$ & $0.031^{*}$ & $1.40(1.03-1.90)$ \\
\hline Allelic frequency & $267(67)$ & $295(74)$ & & \\
C & 400 & 400 & & \\
T & $13(6.5)$ & $5(2.5)$ & 0.054 & $2.71(0.95-7.75)$ \\
Total & $187(93.5)$ & $195(97.5)$ & & \\
\hline Recessive model & 200 & 200 & & \\
CC & $120(60)$ & $100(50)$ & $0.044^{*}$ & $1.50(1.01-2.23)$ \\
$\quad$ TC+TT & $80(40)$ & $100(50)$ & & \\
Total & 200 & 200 & & \\
\hline Dominant model & & \\
TC+CC & & & \\
$\quad$ TT & Total &
\end{tabular}

A Chi square test was applied to calculate" $p$ " value, Odds ratio (OR) and confidence interval (Cl). $A$ "p"of $<0.05$ is statistically significant.

\section{RESULTS}

Out of total 400 subjects, 200 were the cases of Met S and 200 controls. Of these, $320(80 \%)$ were males and $80(20 \%)$ were the females. The mean \pm SD of the ages of the subjects in cases and controls were $46.92 \pm 8.06$ and $46.80 \pm 8.04$ years, respectively. There was no significant difference in mean ages of the two groups $(p$ $=0.877$ ). Median duration of Met $S$ was 4 (2-4) years. Subjects with Met $S$ had significantly higher waist circumference, BP, serum lipid and glycemic parameters compared to the controls $(p<0.001$, Table I).

In total study population, the observed frequency of the dominant (wild) genotype TT was 180 (45\%), TC (heterozygous) $202(50.5 \%)$ and CC (recessive/minor genotype) 18 (4.5\%). Genotypes deviated from HardyWeinberg equilibrium. On comparison of the allelic and genotypic models in two groups, minor allele $C$ of APOA5-1131T $>C$ was significantly higher in Met $S$ as compared to the controls $(p=0.031)$. Frequency of the wild genotype TT was $80(40 \%)$, and $100(50 \%)$ in cases and controls; whereas, frequency of the minor genotype CC in cases and controls was $13(6 \%)$ and $5(2 \%)$, respectively. The frequency of the heterozygous TC in cases and controls was 107 (54\%) and 95 (48\%). There was significant difference of the genotype frequencies in the two groups $(p=0.039)$. In the Dominant model $(\mathrm{TC}+\mathrm{CC}$ vs. TT), frequency of the genotype ' $\mathrm{TC}+\mathrm{CC}$ ' was significantly higher in cases than the controls $(p=0.044)$. There was no significant difference of the genotype frequencies of the recessive model (CC vs. TC+TT) in two groups ( $p=0.054$, Table II).

Logistic regression analysis was applied to see the association of the dominant $(\mathrm{TC}+\mathrm{CC}$ vs. TT) and recessive models (CC vs. TC+TT) of APOA5-1131T >C with Met $S$ after controlling for the confounders such as age and sex. In the dominant model (TC+CC vs. TT) $\mathrm{TC}+\mathrm{CC}$ genotype was significantly associated with the increased risk of Met $S(p=0.044 ; O R=1.50 ; \mathrm{Cl}=1.01$ 2.23) as compared to the TT genotype and the association remained significant after controlling for age and sex $(p=0.043)$. There was no significant association of the genotypes in the recessive model with the Met $S(p=0.06)$.

All the Met $S$ related traits such as BP, waist circumference, serum triglycerides, HDL-c and serum glucose

Table III: Comparison of Met S related traits in dominant genotype model of APOA5-1131T>C.

\begin{tabular}{|c|c|c|c|c|c|c|}
\hline \multirow[t]{2}{*}{ Study parameters } & \multicolumn{3}{|c|}{ Metabolic syndrome } & \multicolumn{3}{|c|}{ Healthy group } \\
\hline & TT & $\mathrm{TC}+\mathrm{CC}$ & $p$-value & TT & $\mathrm{TC}+\mathrm{CC}$ & $\mathrm{p}$-value \\
\hline Systolic BP & $130(110-130)$ & $130(120-140)$ & 0.099 & $115(110-120)$ & $110(110-125)$ & 0.233 \\
\hline Diastolic BP & $80(70-90)$ & $80(70-90)$ & 0.126 & $80(70-80)$ & $80(70-80)$ & 0.642 \\
\hline Waist circumference & $96(91-102)$ & $104(98-108)$ & $<0.001^{*}$ & $79.5(70-85)$ & 85 (69.25-94.5) & $0.022^{*}$ \\
\hline Serum triglycerides & $175(133-249)$ & 201.5 (148-297) & $0.043^{*}$ & $128(105.5-149.5)$ & 148 (124.45-169) & $0.006^{*}$ \\
\hline Serum HDL & $38.5(32-44.75)$ & $38(31-44.75)$ & 0.659 & $39(37-43)$ & 39 (36.75-42.25) & 0.730 \\
\hline Serum glucose & $8(6-11.27)$ & $8.13(6.57-10.63)$ & 0.556 & $5.3(4.5-5.8)$ & $4.97(4.04-5.3)$ & 0.985 \\
\hline HOMA-IR & $8.35(4.81-14.8)$ & $11(6.07-18.75)$ & 0.205 & $2.66(1.59-6.89)$ & $1.99(1.33-2.93)$ & 0.285 \\
\hline
\end{tabular}

Values given are Median (IQR) as related to the results obtained through the Shapiro-Wilk's statistics. " $p$ " value is generated by Mann Whitney $U$ test. A p of less than 0.05 is statistically significant. HOMA-IR = Fasting serum glucose $x$ Fasting serum insulin $/ 22.5$ 


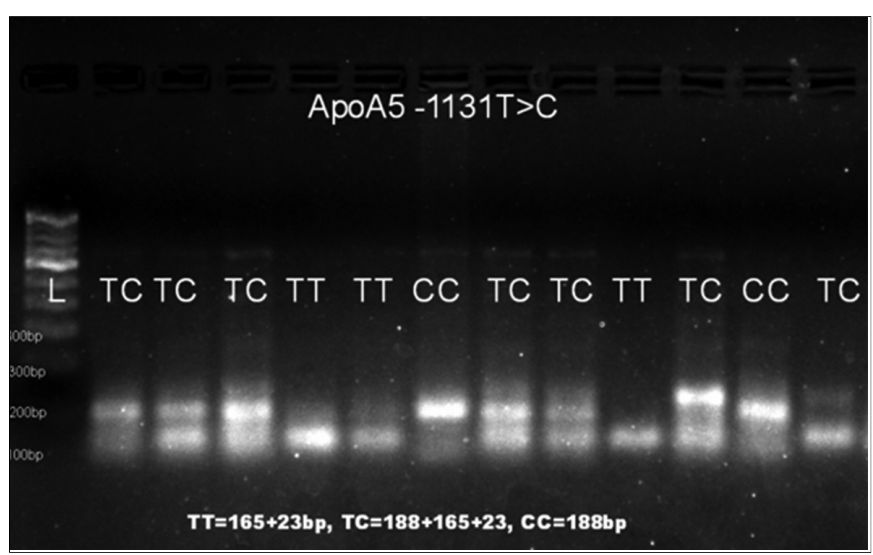

Figure 1: Genotypes of APOA5-1131T>C on Gel electrophoresis. DNA ladder 100base pair:

TT genotype (165- and 23- base pair): CC genotype (188 base pair); TC genotype (188-, 165- and 23- base pair).

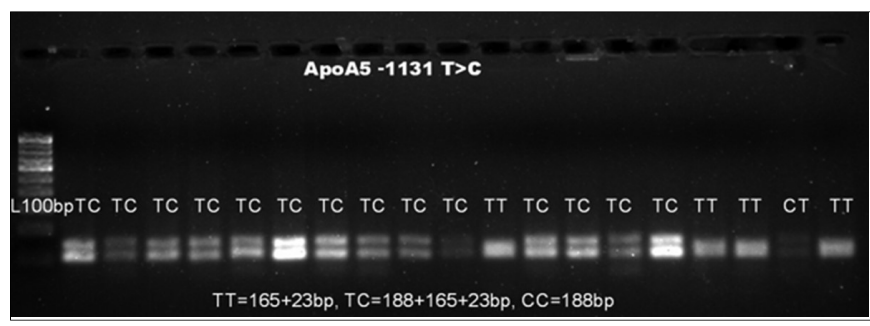

Figure 2: Genotypes of APOA5-1131T $>C$ on Gel electrophoresis. DNA ladder 100base pair:

TT genotype (165- and 23- base pair): TC genotype (188-, 165- and 23- base pair).

were compared in the dominant $(\mathrm{TC}+\mathrm{CC}$ vs. TT) and recessive (CC vs. TC+TT) genotype models of APOA5 $1131 \mathrm{~T}>\mathrm{C}$. Waist circumference and serum triglycerides were significantly higher in $\mathrm{TC}+\mathrm{CC}$ genotype as compared to the TT in Dominant APOA5 genetic model in Met $S$ and healthy group. There was no significant difference regarding BP, HDL and HOMA-IR between the two groups (Table III). On comparison of these parameters in the recessive genotype model (CC vs. $\mathrm{TC}+\mathrm{TT}$ ) serum triglycerides were significantly higher in CC variant as compared to the TC+TT in Met $S$ and healthy group $(p=0.007$ and $<0.001)$. There was no significant difference of the other parameters between the two groups.

\section{DISCUSSION}

In the present study, the minor ' $C$ ' allele of APOA5 $1131 \mathrm{~T}>\mathrm{C}$ variant was found to be associated with the increased risk of Met $S$ (Odds ratio; 1.40 , $p$ value $0.031^{*}$ ) as compared to the wild ' $T$ ' allele. In a previous study, on patients of acute myocardial infarction, the minor allele frequency of APOA5-1131T $>C$ was reported to be considerably higher in Pakistanis as compared to the Germans ( 0.17 vs. 0.07$)$ and it was also significantly associated with the plasma triglyceride concentration. ${ }^{12}$ However, in another study on Pakistanis with coronary artery disease (CAD)' $C$ ', allele of APOA5 -1131T>C variant was found to be associated with the increased triglyceride levels and not with the CAD. ${ }^{11}$ In the present study, minor allele frequency (MAF) of APOA5 -1131T >C was found to be 0.29 ; comparable to the MAF of APOA5 $-1131 \mathrm{~T}>\mathrm{C}$ in Koreans and Chinese $(0.29$ and 0.30 respectively) while it was considerably higher than that in the Europeans and Germans (0.09 and 0.07), respectively. ${ }^{18}$ The ' $C$ ' variant of $-1131 \mathrm{~T}>\mathrm{C}$ was reported to be associated with the increased risk of Met $S$ and also with the increased blood triglyceride levels in Korean and Chinese population. ${ }^{19,20}$ The minor C allele was also found to be a risk factor for early onset myocardial infarction and raised serum triglyceride levels in Italian population. ${ }^{21}$ The 'C' variant of APOA5 $1131 \mathrm{~T}>\mathrm{C}$ was reported to be associated with hypertriglyceridemia in Ashkenazi and Yemenite jews but not in Sephardic. 22 In Farmingham offspring study, increase in BMI and weight gain after fatty meal intake was considerably higher in the major " $\mathrm{T}$ " allele carriers as compared to those with the " $\mathrm{C}$ " allele. ${ }^{23}$ In addition to the established risk factors, ethnic and inter-individual genetic variations might predispose to the metabolic derangements resulting from complex heterogenous cluster of insulin resistance and the related traits.

In this study, waist circumference and fasting triglycerides were found to be significantly higher in the ' $T C+C C$ ' genotype of APOA5 -1131T>C as compared to the 'TT'. This difference was observed in Met $S$ group and in healthy group as well. Met $S$ also named as inflammatory syndrome, is characterised by adipose dysfunction, central or visceral adiposity and dyslipidemia. The increase in waist circumference and triglyceride levels in the variant genotype of APOA5 in Met S might be due to the confounding inflammatory state, but the difference was observed in the healthy group as well. These results were in concordance with the previous studies on Pakistanis, Germans and Koreans reporting association of APOA5 promoter polymorphism with plasma lipid profile.11,12,18 The possible bridge between the APOA5-1131T $>C$ polymorphism and deranged lipid profile is the protein APOA5; this promoter polymorphism might suppress APOA5 expression as reported in a previously published double blind multicenter study on type 2 diabetic men and women.24 However, in another study, on EPIC Norfolk cohort $1131 \mathrm{~T}>\mathrm{C}$ promoter polymorphism was found to be associated with $C A D$ and triglyceride levels but not with the low APOA5 levels; the association between APOA5 and serum triglycerides was explained on the basis of APOAC3 linkage disequilibrium. 25 These equivocal results suggest that further work is required to see the role of this genetic variant in dyslipidemias, insulin resistance and associated disorders. There are certain limitations of the present study as plasma APOA5 levels were not determined, and majority of the patients of Met $S$ were on anti-diabetic, antihypertensive and lipid lowering medications. These medicines interfere 
with the normal metabolic homeostasis. However, the control group was not taking any medication and waist circumference and serum triglycerides were significantly higher in the ' $\mathrm{TC}+\mathrm{CC}$ ' variant of APOA5 in the controls as well.

\section{CONCLUSION}

There was significant association of the TC+CC genotype of the APOA5 promoter polymorphism with Met $S$ after controlling for the confounders such as age and gender. Waist circumference and serum triglycerides were significantly higher in the "TC+CC" genotype of APOA5 $-1131 \mathrm{~T}>\mathrm{C}$ as compared to the "TT" in cases of Met $\mathrm{S}$ and in controls. Further work is required to explore the role of this genetic variant in metabolic derangements and dyslipdemias associated with Met $\mathrm{S}$.

Disclosure: This article is part of $\mathrm{PhD}$ thesis of the first author.

\section{REFERENCES}

1. Kaur J. A comprehensive review on metabolic syndrome. Cardiol Res Pract 2014; 2014:943162.

2. Gami AS, Witt BJ, Howard DE, Erwin PJ, Gami LA, Somers VK, et al. Metabolic syndrome and risk of incident cardiovascular events and death: A systematic review and meta-analysis of longitudinal studies. J Am Coll Cardiol 2007; 49:403-14.

3. Ranasinghe $P$, Mathangasinghe $Y$, Jayawardena $R$, Hills $A$, Misra A. Prevalence and trends of metabolic syndrome among adults in the Asia-Pacific region: A systematic review. BMC Public Health 2017; 17:101.

4. Xu C, Bai R, Zhang D, Li Z, Zhu H, Lai M, et al. Effects of APOA5 -1131T >C (rs662799) on fasting plasma lipids and risk of metabolic syndrome: Evidence from a case-control study in China and a meta-analysis. PloS One 2013; 8:e56216.

5. Hubácek JA, Adámková V, Vrablík M, Kadlecová M, Zicha J, Kunes $\mathrm{J}$, et al. Apolipoprotein A5 in health and disease. Physiol Res 2009; 58:S101-S9.

6. O'Brien PJ, Alborn WE, Sloan JH, Ulmer M, Boodhoo A, Knierman MD, et al. The novel apolipoprotein A5 is present in human serum, is associated with VLDL, HDL, and chylomicrons, and circulates at very low concentrations compared with other apolipoproteins. Clin Chem 2005; 51:351-9.

7. Pennacchio LA, Rubin EM. Apolipoprotein A5, a newly identified gene that affects plasma triglyceride levels in humans and mice. Arterioscler Thromb Vasc Biol 2003; 23: 529-34.

8. Song KH, Cha S, Yu S-G, Yu H, Oh SA, Kang NS. Association of apolipoprotein A5 gene 1131TC polymorphism with the risk of metabolic syndrome in Korean subjects. BioMed Res Int 2013; 2013

9. Xia J, Cai W, Peng C. Association of APOA5 T1131C polymorphism and risk of coronary artery disease. Int J Clin Exp Med 2015; 8:8986-94.

10. Mattei J, Demissie S, Tucker KL, Ordovas JM. Apolipoprotein A5 polymorphisms interact with total dietary fat intake in association with markers of metabolic syndrome in Puerto Rican older adults. J Nutr 2009; 139:2301-8.

11. Shahid SU, Shabana N, Cooper JA, Rehman A, Humphries $\mathrm{SE}$. Common variants in the genes of triglyceride and HDL-C metabolism lack association with coronary artery disease in the Pakistani subjects. Lipids Health Dis 2017; 16:24.

12. Saleheen D, Soranzo N, Rasheed A, Scharnagl H, Gwilliam R, Alexander $\mathrm{M}$, et al. Genetic determinants of major blood lipids in Pakistanis compared with Europeans. Circ Cardiovasc Genet 2010; 3:348-57.

13. Alberti KGMM, Zimmet P, Shaw J. Metabolic syndrome - a new world-wide definition. A consensus statement from the international diabetes federation. Diabet Med 2006; 23:469-80.

14. Association AD. Diagnosis and classification of diabetes mellitus. Diabetes Care 2010; 33 (Suppl 1):S62-9.

15. Ferreira-Hermosillo A, Molina-Ayala M, Ramírez-Rentería C, Vargas G, Gonzalez B, Isibasi A, et al. Inflammatory cytokine profile associated with metabolic syndrome in adult patients with type 1 diabetes. J Diabetes Res 2015; 2015:972073.

16. Matthews D, Hosker J, Rudenski A, Naylor B, Treacher D, Turner R. Homeostasis model assessment: insulin resistance and beta-cell function from fasting plasma glucose and insulin concentrations in man. Diabetologia 1985; 28:412-9.

17. Yin R-X, Li Y-Y, Lai C-Q. Apolipoprotein A1/C3/A5 haplotypes and serum lipid levels. Lipids Health Dis 2011; 10:140.

18. Son KY, Son HY, Chae J, Hwang J, Jang S, Yun JM, et al. Genetic association of APOA5 and APOE with metabolic syndrome and their interaction with health-related behavior in Korean men. Lipids Health Dis 2015; 14:105.

19. Kim YR, Hong S-H. Association of apolipoprotein A5 gene polymorphisms with metabolic syndrome in the Korean population. Genet Test Mol Biomarkers 2016; 20:130-6.

20. Ong KL, Jiang CQ, Liu B, Jin YL, Tso AW, Tam S, et al. Association of a genetic variant in the apolipoprotein $\mathrm{A} 5$ gene with the metabolic syndrome in Chinese. Clin Endocrinol (Oxf) 2011; 74:206-13.

21. De Caterina R, Talmud PJ, Merlini PA, Foco L, Pastorino R, Altshuler D, et al. Strong association of the APOA5-1131T> C gene variant and early-onset acute myocardial infarction. Atherosclerosis 2011; 214:397-403.

22. Ken-Dror G, Goldbourt U, Dankner R. Different effects of apolipoprotein A5 SNPs and haplotypes on triglyceride concentration in three ethnic origins. J Hum Genet 2010; 55: 300-7.

23. Corella D, Lai CQ, Demissie S, Cupples LA, Manning AK, Tucker $\mathrm{KL}$, et al. APOA5 gene variation modulates the effects of dietary fat intake on body mass index and obesity risk in the Framingham Heart Study. J Mol Med (Berl) 2007; 85:119-28.

24. Dallinga-Thie GM, van Tol $A$, Hattori $H$, van Vark-van der Zee LC, Jansen $\mathrm{H}$, Sijbrands EJ, et al. Plasma apolipoprotein A5 and triglycerides in type 2 diabetes. Diabetologia 2006; 49:1505-11.

25. Vaessen SF, Schaap FG, Kuivenhoven JA, Groen AK, Hutten BA, Boekholdt SM, et al. Apolipoprotein AV, triglycerides and risk of coronary artery disease: The prospective Epic-Norfolk population study. J Lipid Res 2006; 47:2064-70. 